INTEGRAL POINTS ON A VERY FLAT CONVEX CURVE

Jean-Marc DESHOUILLERS (corresponding author)

Institut Mathématique de Bordeaux, UMR 5251

Université de Bordeaux, CNRS, Bordeaux INP

33405 TALENCE Cedex

France

jean-marc.deshouillers@math.u-bordeaux.fr

Georges GREKOS

Institut Camille Jordan, UMR 5208

Université de Saint-Etienne (membre de l'Université de Lyon), CNRS

23 rue du Dr Paul Michelon

42023 SAINT-ÉTIENNE

France

grekos@univ-st-etienne.fr

Subject classification number(s): 11H06

Keywords: geometry of numbers, integer points, strictly convex curves.

Acknowledgements: Jean-Marc Deshouillers acknowledges the support of the binational research project MuDeRa, funded by the French and Austrian Science Funds ANR and FWF. The research of Georges Grekos has been supported by the French grant CAESAR ANR-12-BS01-0011.

Abstract:

The second named author studied in 1988 the possible relations between the length $\ell$, the minimal radius of curvature $r$ and the number of integral points $N$ of a strictly convex flat curve in $\mathbb{R}^{2}$, stating that $N=O\left(\ell / r^{1 / 3}\right)(*)$, a best possible bound even when imposing the tangent at one extremity of the curve; here flat means that one has $\ell=r^{\alpha}$ for some $\alpha \in[2 / 3,1)$. He also proved that when $\alpha \leq 1 / 3$, the quantity $N$ is bounded. In this paper, the authors prove that in general the bound $(*)$ cannot be improved for very flat curves, i.e. those for which $\alpha \in(1 / 3,2 / 3)$; however, if one imposes a 0 tangent at one extremity of the curve, then $\left.{ }^{*}\right)$ is replaced by the sharper inequality $N \leq \ell^{2} / r+1$. 


\title{
INTEGRAL POINTS ON A VERY FLAT CONVEX CURVE
}

\author{
JEAN-MARC DESHOUILLERS, GEORGES GREKOS
}

To Krishnaswami Alladi, for his 60th birthday

\begin{abstract}
The second named author studied in 1988 the possible relations between the length $\ell$, the minimal radius of curvature $r$ and the number of integral points $N$ of a strictly convex flat curve in $\mathbb{R}^{2}$, stating that $N=$ $O\left(\ell / r^{1 / 3}\right)\left(^{*}\right)$, a best possible bound even when imposing the tangent at one extremity of the curve; here flat means that one has $\ell=r^{\alpha}$ for some $\alpha \in[2 / 3,1)$. He also proved that when $\alpha \leq 1 / 3$, the quantity $N$ is bounded. In this paper, the authors prove that in general the bound $\left(^{*}\right)$ cannot be improved for very flat curves, i.e. those for which $\alpha \in(1 / 3,2 / 3)$; however, if one imposes a 0 tangent at one extremity of the curve, then $\left(^{*}\right)$ is replaced by the sharper inequality $N \leq \ell^{2} / r+1$.
\end{abstract}

\section{INTRODUCTION}

In 1926, V. Jarník [2] started the study of integral points on general strictly convex curves in the euclidean plane, showing that the number of points of such a curve of length $\ell$ cannot be larger than $(C+o(1)) \ell^{2 / 3}$ (where $C=3(16 \pi)^{-1 / 3}$ ) and that this bound is optimal. The second named author [1] considered in 1988 the case of flat curves, i.e. curves with a radius of curvature significantly larger than their length. Let us present the results of [1], starting with some definitions.

We let $\Gamma$ be a $\mathcal{C}^{2}$ strictly convex curve in the euclidean plane $\mathbb{R}^{2}$. More precisely

$$
\Gamma=\left\{M(t)=(x(t), y(t)) \in \mathbb{R}^{2}: 0 \leq t \leq 1\right\},
$$

where $x$ and $y$ are two $\mathcal{C}^{2}$ functions on $[0,1]$, such that

$$
\forall t \in[0,1]: x^{\prime}(t) y^{\prime \prime}(t)-x^{\prime \prime}(t) y^{\prime}(t) \neq 0 .
$$

We denote the length of $\Gamma$ by $\ell(\Gamma)$; to each point $M$ on $\Gamma$, we associate the radius of curvature of $\Gamma$ at $M$, denoted by $R(M)$, and define the minimal radius of curvature of $\Gamma$ by the relation

$$
r(\Gamma)=\min \{R(M): M \in \Gamma\} ;
$$

notice that (2) implies $r(\Gamma)>0$.

The number of "integral points" on $\Gamma$, i.e. points with coordinates in $\mathbb{Z}^{2}$, is denoted by $N(\Gamma)$. 
Finally, the quantity $\alpha$ is defined by

$$
\alpha=\frac{\log \ell(\Gamma)}{\log r(\Gamma)} .
$$

The main results of [1] (Théorème 1 and Théorème 2) can be rephrased as Theorem 1 and Theorem 2 below.

Theorem 1. If $r \geq 1$ and $\ell \geq r^{1 / 3}$, then, for any curve $\Gamma$ with length $\ell$ and minimal radius of curvature $r$, we have

$$
N(\Gamma) \leq 2 \ell r^{-1 / 3}
$$

The second result shows that this result is best possible, up to the numerical value of the constant, but moreover that one can impose any value for the tangent at the origin of the curve $\Gamma$, determined by the vecteur

$$
T_{0}(\Gamma)=\left(x^{\prime}(0), y^{\prime}(0)\right) \text {. }
$$

Theorem 2. Let $T$ be a non zero vector in $\mathbb{R}^{2}$ and $\alpha \in(2 / 3,1)$. For any $r \geq r_{0}(T, \alpha)$ there exists a curve $\Gamma$ with $T_{0}(\Gamma)=T, \ell(\Gamma)=10^{5} r^{\alpha}$ such that

$$
N(\Gamma) \geq 10^{-6} \ell r^{-1 / 3} \text {. }
$$

A first consequence of Theorem 1 is that for $r \geq 1$ and $\ell \leq r^{1 / 3}$ one has $N(\Gamma) \leq 2$ : simply prolongate the curve with a suitable arc of a circle until its length is $r^{1 / 3}$. This implies that the case $\alpha \in[0,1 / 3]$ is essentially trivial.

But Theorem 2 only deals with the case $\alpha \in(2 / 3,1)$, hence a natural question arises: what happens when $\alpha \in(1 / 3,2 / 3]$ ? It is conjectured in [1] that in this case one has

$$
N(\Gamma)=O\left(\ell(\Gamma)^{2} / r(\Gamma)\right) .
$$

Notice that $l^{2} / r<l / r^{-1 / 3}$ excatly when $\alpha<2 / 3$.

The aim of this note is to show that this conjecture is partially correct, but partially false. More precisely, we have

Theorem 3. Let $\Gamma$ be a strictly convex curve such that $y^{\prime}(0)=0$ and $\ell(\Gamma) \leq$ $r(\Gamma)$. We have

$$
N(\Gamma) \leq\left(\ell(\Gamma)^{2} / r(\Gamma)\right)+1 .
$$

On the other hand, if we do not fix $T_{0}$, Theorem 2 can be extended to any $\alpha>1 / 3$; moreover, we may ask the radius of curvature to keep the same size, up to factor $(1+o(1))$, all over the curve.

Theorem 4. For any $\alpha \in(1 / 3,2 / 3), c_{1}>1$ and $c_{2}<2^{-1 / 3}$ there exists $r_{0}=r_{0}\left(\alpha, c_{1}, c_{2}\right)$ such that for any $r \geq r_{0}$, there exists a strictly convex curve 
$\Gamma$ such that

$$
\begin{aligned}
\forall M \in \Gamma: r(\Gamma)=r & \leq R(M) \leq c_{1} r \\
\ell(\Gamma) & =r^{\alpha} \\
N(\Gamma) & \geq c_{2} \ell(\Gamma) / r(\Gamma)^{1 / 3}
\end{aligned}
$$

\section{Proof of Theorem 3}

We first introduce a technical tool. Lemma 1 is true without assuming the second part of (iii); however we do not see how to prove it without using some clumsy limiting process; in our case of interest, the case of the circle, this limiting process can be easily performed as it is done in Corollary 1.

Lemma 1. Let $a<b$ be two real numbers and $f$ and $g$ be in $\mathcal{C}_{\mathbb{R}}^{2}[a, b]$ with the properties

(i) $\quad f(a) \leq g(a)$ and $f^{\prime}(a)=g^{\prime}(a)$,

(ii) $\forall x \in[a, b]: f^{\prime \prime}(x)>0$ and $g^{\prime \prime}(x)>0$,

(iii) $\forall x \in[a, b]: r_{f}(x) \geq r_{g}(x)$ and $r_{f}(a)>r_{g}(a)$,

where $r_{f}(x)$ denotes the radius of curvature of the graph of $f$ at the point $(x, f(x))$.

Then, for all $x \in[a, b]: f(x) \leq g(x)$.

Proof. We first prove the following

$$
\forall x \in(a, b): f^{\prime}(x)<g^{\prime}(x) .
$$

We recall that we have

$$
r_{f}(x)=\frac{\left(1+f^{\prime}(x)^{2}\right)^{3 / 2}}{\left|f^{\prime \prime}(x)\right|} .
$$

Thanks to (i) and (iii) we have $f^{\prime \prime}(a)<g^{\prime \prime}(a)$ and there exists $c \in(a, b]$ such that $f^{\prime}(x)<g^{\prime}(x)$ for all $x \in(a, c)$; we choose $c$ to be maximal and prove by contradiction that $c=b$, which proves (12). If $c<b$, we have $f^{\prime}(c)=g^{\prime}(c)$; by Rolle's theorem, there exists $d \in(a, c)$ with $f^{\prime \prime}(d)=g^{\prime \prime}(d)$; but $f^{\prime}(d)<g^{\prime}(d)$, which implies $r_{f}(d)<r_{g}(d)$, a contradiction.

From (12), we get for $x \in(a, b)$ :

$$
f(x)=f(a)+\int_{a}^{x} f^{\prime}(t) d t \leq g(a)+\int_{a}^{x} g^{\prime}(t) d t=g(x) .
$$

We state a corollary of this lemma which will be more convenient in the sequel. 
Corollary 1. Let $R>0, a<b \leq a+R$ and $f \in \mathcal{C}_{\mathbb{R}}^{2}[a, b]$ be such that

(i) $\quad f^{\prime}(a)=0$

(ii) $\forall x \in[a, b): f^{\prime \prime}(x)>0$,

(iii) $\quad \forall x \in[a, b): r_{f}(x) \geq R$.

Then, for all $x \in[a, b]: f(a) \leq f(x) \leq f(a)+R-\sqrt{R^{2}-(x-a)^{2}}$.

Proof. For the lower bound, notice that $f$ is convex and thus above its tangent at the point 0 . For the upper bound, apply Lemma 1 on the interval $[a, a+R-2 / N]$ with functions $f$ and

$$
g_{N}(x)=f(a)+R-1 / N-\sqrt{(R-1 / N)^{2}-(x-a)^{2}}
$$

for large $N$; notice that the graph of $g_{N}$ is an arc of the circle with radius $R-1 / N$ and centre $(a, f(a)+R-1 / N)$.

We now prove Theorem 3 .

Proof. Let $\Gamma$ be given by (1) with $y^{\prime}(0)=0$; by $(2)$ we have $x^{\prime}(0) \neq 0$ and $y^{\prime \prime}(0) \neq 0$. Without loss of generality, we may assume $x^{\prime}(0)>0$ and $y^{\prime \prime}(0)>0$, since changing the sign of $x$ or that of $y$ or both correspond to symmetries which do not change neither the length of the curve, nor its radius of curvature, nor the number of its integral points. Furthermore, when $\ell \leq r$, as we assumed, the curve $\Gamma$ may be seen as the graph of a function $f_{\Gamma}$ defined on $[x(0), x(1)]$. Brief explanation: if we denote by $s$ the curviligne absissa on $\Gamma$ and $\theta$ the angle between the absissa axis and the tangent to $\Gamma$, we have the relation $s^{\prime}=R \theta^{\prime}$; since at each point of $\Gamma$ the radius of curvature of $\Gamma$ is at least $r=r(\Gamma)$, we have $s^{\prime} \geq r \theta^{\prime}$; integrating this relation, we get that at each point of the curve we have $\theta \leq \ell / r \leq 1<\pi / 2$. We now apply Corollary 1 to the function $f_{\Gamma}$, with $R=r(\Gamma), a=x(0), b=x(1)$; we have $b-a=x(1)-x(0)=\int_{0}^{1} \sqrt{x^{\prime}(t)^{2}} d t \leq$ $\ell \leq R$; thus, for any $x \in[a, b]$ we have $f(x) \in\left[f(a), f(a)+R-\sqrt{R^{2}-\ell^{2}}\right]$, an interval with length at most $R-R \sqrt{1-(\ell / R)^{2}} \leq \ell^{2} / R$, which contains at most $\ell^{2} / R+1$ integral points. The number of integral points on $\Gamma$ is at most the number of points with integral ordinates, and since the function $f_{\Gamma}$ is increasing, we have (8).

\section{Proof of Theorem 4}

Proof. Let $\alpha \in(1 / 3,2 / 3)$. For given $r \geq 1$ we let $X$ and $H$ be defined by

$$
\frac{\left(1+4 X^{2}\right)^{3 / 2}}{2}=r \text { and } \int_{X}^{X+H}\left(1+4 x^{2}\right)^{1 / 2} d x=r^{\alpha} .
$$

We easily check that we have

$$
X \sim 4^{-1 / 3} r^{1 / 3} \text { and } H \sim 2^{-1 / 3} r^{\alpha-1 / 3}=o(X), \text { as } r \rightarrow \infty .
$$

We consider for $\Gamma$ the graph of the function $f$ defined by

$$
\forall x \in[X, X+H]: f(x)=x^{2} .
$$


By (14) and (15), at any point $M=(x, f(x))$, we have

$$
r=\frac{\left(1+4 X^{2}\right)^{3 / 2}}{2} \leq \frac{\left(1+4 x^{2}\right)^{3 / 2}}{2}=R(M) \leq \frac{\left(1+4(X+H)^{2}\right)^{3 / 2}}{2} \sim r,
$$

which implies (9). We also have

$$
\ell(\Gamma)=\int_{X}^{X+H}\left(1+4 x^{2}\right)^{1 / 2}=r^{\alpha},
$$

which is (10). We finally have

$$
N(\Gamma)=\operatorname{Card}([X, X+H] \cap \mathbb{Z}) \sim H \sim 2^{-1 / 3} r^{\alpha-1 / 3},
$$

which implies (11).

\section{REFERENCES}

[1] G. Grekos. - Sur le nombre de points entiers d'une courbe convexe, Bull. Sci. Math., 112 (1988), 235-254.

[2] V. Jarník. - Über die Gitterpunkte auf konvexen Kurven, Math. Zeitschrift, 24 (1926), 500-518. 